\title{
Design of Multicolour LED with Control and Monitoring System for Plant Growth
}

\author{
Ng Wei Choon ${ }^{1}$, Nurul Amziah Md Yunus ${ }^{1,2,}$, Izhal Abdul Halin ${ }^{1,2}$ \\ ${ }^{1}$ Department of Electrical and Electronic Engineering, Faculty of Engineering, Universiti Putra Malaysia, 43400 Serdang UPM, \\ Selangor. Malaysia. \\ ${ }^{2}$ Advanced Material Synthesis and Fabrication Laboratory (AMSF), Faculty of Engineering, Universiti Putra Malaysia, 43400 Ser- \\ dang UPM, Selangor. Malaysia.
}

\begin{abstract}
The aim of this work is to create an artificial environment LED based for plants where the goal is to control LED lighting and monitor plant growth. This will contribute on issue like food production. This work involves the use of a web development microcontroller kit, which will help to make online or loT (Internet of Things) project and design easy. The microcontroller will come with an integrated $\mathrm{Wi}-\mathrm{Fi}$ to collect and control variable parameter and also programming language to develop graphical user interface (GUI). It is expected that the plant growth environment can be monitored and controlled or set through cloud via Wi-Fi on the board. Multicolour light emitting diode (LED) lamps are used. This system will improve the performance of available plant growth area where it will become more flexible in term of providing artificial environment with a better supervision.
\end{abstract}

\section{Introduction}

There are some essential criteria for plant to grow well such as having a good surrounding temperature, soil moisture, surrounding humidity and light intensity. This is a design of plant growth multicolour LED based controller and monitoring system where an artificial environment includes parameters such as temperature, soil moisture, humidity and light intensity is prepared conducively for the plant growth. These parameters are under supervision through detail control by getting the information through designed graphical user interface (GUI) in PC or mobile application. The main component is the lighting source where its effect to the plant growth will be studied.

LED is used because it has low power consumption, generates less heat and has multiple colours [1]. Different colours of LEDs bring different effects to the plant growth. There are few types of visible light includes ultraviolet, violet, blue, green, yellow, red and far red. Some of the light might harm the growth of plant such as ultraviolet light. Exposure to ultraviolet light will retard the plant growth. For other band of visible light, violet light can enhances the colour, taste and aroma of plants. Blue, green, yellow, red and far red can improve the growth of plants in different aspect. These lights have different wavelength and energy level. Certain plants can yields more leaves and crops by combining red and blue light. Besides that, green light can enhance the chlorophyll production [2]. In most past researches, blue and red light shows the most significant improvement for the plant growth [3-

\footnotetext{
* Corresponding author: amziah@upm.edu.my
}

5]. Hence, in this paper, multicolour LEDs focusing on the red and blue light colours are to be discussed for better plant growth.

As well known, plant growth is sunlight and weather dependent. Both sunlight and weather are intermittent. Crop production will be limited due to weather issue. For example, if there is natural disaster such as flood after few days raining, it will cause shortage of vegetables in the market and price rises because production cannot meet the demand. Moreover, plants need caring cares where there is need to make sure the plants gain sufficient water, nutrition and sunlight. For a large scale farm, these works are burden to farmers if only a few number of farmers are working. Furthermore, close distance monitoring is needed to make sure the plants grow well.

The main objective of this work is to create an artificial light environment with other controlling parameters for plant growth. Second, is to implement the plant monitoring and controlling system by using Raspberry Pi. Third, is to enable far distance real time monitoring.

\section{System and Structure}

The overview of entire system is shown in Figure 1. This system is divided into five parts: artificial light environment, main controller, sensors, actuators and user interface. Artificial light environment will be implemented by using common anode 4-pins LED strip. Common anode pin is connected to $12 \mathrm{~V}$ supply and cathode pin of RGB will be connected to ground 
in order to light them up. For example, to light up red LED, cathode of $\mathrm{R}$ is grounded.

For main controller, Raspberry $\mathrm{Pi}$ model $\mathrm{B}+$ is used. Basically, the GPIO pins of the Raspberry Pi will be fully utilized in this system. The sensors and actuators will be connected to these GPIO pins to read or write the digital signal. Python will be used to program and interface the sensors part and actuators part so that the sensors and actuators are working together to maintain the environmental parameters at optimum level. Besides that, Raspberry Pi will also act as the web server where the data obtained from the sensors can be stored and transmitted to a website.

As for the sensors part, DHT11 isused to measure the surrounding temperature and humidity while soil moisture sensor is to detect the presence of water in the soil. Ultrasonic sensor and webcam are used to monitor the plant growth where ultrasonic sensor can detect the height of the plant whereas the webcam will capture the video of the plant. The water pump as one of the actuators is used for watering and producing the mist to increase the humidity. Fan is used to decrease the humidity while peltier is used to either warm up or chill down the surrounding temperature. For most of the actuators used, the required voltage is $12 \mathrm{~V}$ but the voltage supply pins of the Raspberry Pi are only $3.3 \mathrm{~V}$ and $5 \mathrm{~V}$. Therefore the relay is used to receive signal from Raspberry Pi and turn on or off the secondary circuit. The actuators will be connected to the external $12 \mathrm{~V}$ voltage supply and to the output of relay. GPIO pin of the Raspberry Pi is connected to the input pin of the relay. Hence, GPIO pin can determine whether the relay to switch on or off the secondary circuit with actuators.

For the user interface, a website and mobile application are developed to ease the user to display the real time data from the plant. Besides that, the user can always notify the condition of the plant.

The growth of plant will then be monitored through the system. A sample from the type of vegetable plant, apple mint, is chosen and will be planted within this system. Apple mint is a perennial vegetable, herb plant that grows tall and is sometimes called the woolly mint. It is a member of the mint genus Mentha. This herb is generally grown as a culinary herb or ground cover. The mint leaves contains nutrients like iron, potassium, calcium, vitamin $\mathrm{A}$ and $\mathrm{C}$, which replenish our body and alternative of antibiotics. The flowers can be used to make tea which if consumed promotes digestion, cures many ailments such as intestine problems, stomach pain and refreshes the mind [6].

The vegetative growth of the plants will be compared. The number of leaves, the height of the plants and the colour of the leaves will be compared. It is predicted that the vegetable can be harvested in shorter period by using this system and have better growth by using this system.

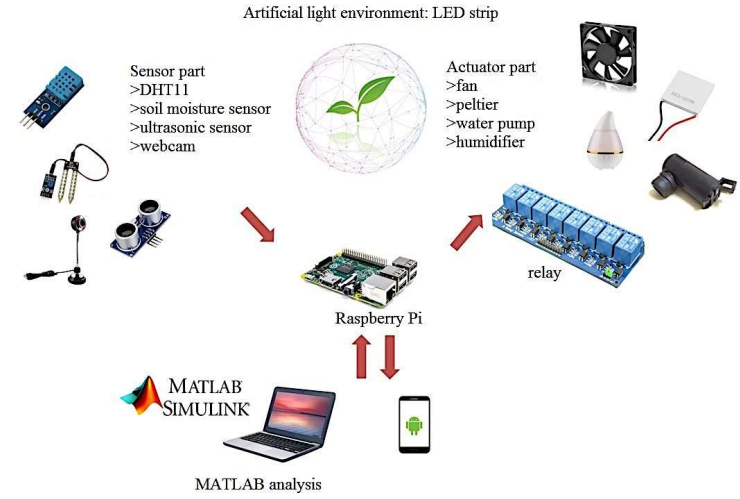

Fig. 1. Overview of the system.

\section{Hardware Development}

As mentioned earlier, Raspberry Pi B+a computer in sized of the credit card is used. The entire system designed is controlled by Raspberry Pi. Besides that, Raspberry Pi also acts as local web server for webcam and sensor data. The webcam video and sensor data can be viewed online in local network.

The artificial light source is common anode 4-pins LED strip and manipulating the input voltage to the 4pins can change its colour. A desired colour can be set for the LED strip by always supply $12 \mathrm{~V}$ to the common anode pin and ground the desired RGB cathode pin. Some colours require two or three RGB cathode pins to be grounded in order to have the desired colour of light-to-light up. For example, purple is combination of red and blue light. Both $\mathrm{R}$ and $\mathrm{B}$ cathode pins should be grounded in order to get the purple light from the LED strip.

Each sensor has its own way to be configured. For examples, there are four pins for ultrasonic sensor, which are ground, Vcc, trigger and echo. Ground pin is grounded and $\mathrm{Vcc}$ pin is connected to a $3.3 \mathrm{~V}$ voltage source of Raspberry Pi as the power supply. Pulse signal will be generated at the GPIO pin of the Raspberry Pi using Python. Consequently, the ultrasonic sensor can emit an ultrasonic wave. When the wave meets an obstacle, it will reflect back and ultrasonic sensor can detect it and send signal to the Raspberry Pi through the echo pin. The time taken for the wave to get back/reflect to the ultrasonic sensor is used to multiply with the speed of wave and divided by 2 to determine the distance. All the hardware will be controlled by the Raspberry Pi, which acts as the main controller.

\section{Software Development}

Python is the main software part for entire system. Python is used to program all the sensors and actuators. By taking the ultrasonic sensor as example, the calculation is needed in order to show the distance. The calculation is programmed in Python and the distance will be shown. Besides that, the data obtained from the sensors will be stored to the MySQL database in the Raspberry Pi. Python can be used to 
store the data into the MySQL database. Moreover, if else statement is also written in Python for the actuators to be turned on or off to maintain the environmental parameters at optimum level which essential for plant growth.

A website is developed so that users can easily view the data of environmental parameters of the plant and the condition of the plant. The website will be developed in HTML and PHP. A login page will be made first to prompt the user to key in their id and password. The id and password are stored in the MySQL database where PHP script will compare the id and password. Only the authorized users can view the plant data. The website is created in local network where user can view the sensors data and live stream video of the plant in local network.

Blynk is used to enable sensor data online not only for local network. This mobile application will obtain the sensor data and display the data to the user when the smart phone is connected to internet.

Matlab and Simulink are used to analyze the data obtained. For example, Matlab function is used for colour segmentation for the artificial light environment. Mean RGB level and mean gray level of few artificial light environments are determined to identify the colour composition in the particular artificial light environment.

\section{Results and Discussion}

Two apple mints have been grown in the designed chamber with the proposed system (illumination of artificial light environment) and outdoor with illumination of sunlight. The duration taken for the experiment is 24 days.

For the artificial light environment, the composition of red and blue light is used where it is proven that red and blue light bring significant plant growth as also highlighted by other researchers [3-5, 7-8]. In order to verify the colours, Matlab was used to carry out colour segmentation to the created artificial light environment. Figure 2 shows the artificial light environment has high level of red and blue level (the top two lines, the most upper is red and just below is blue).

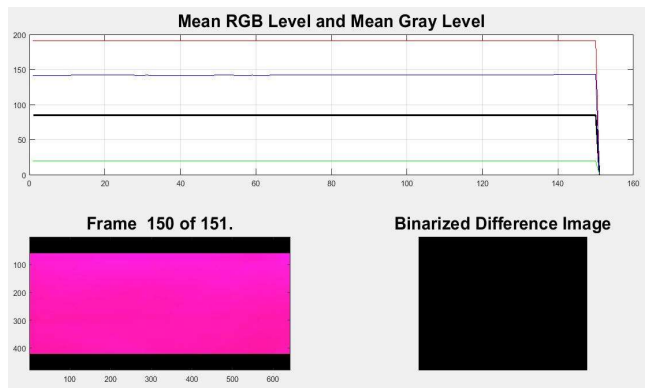

Fig. 2. Colour segmentation of the created artificial light environment.

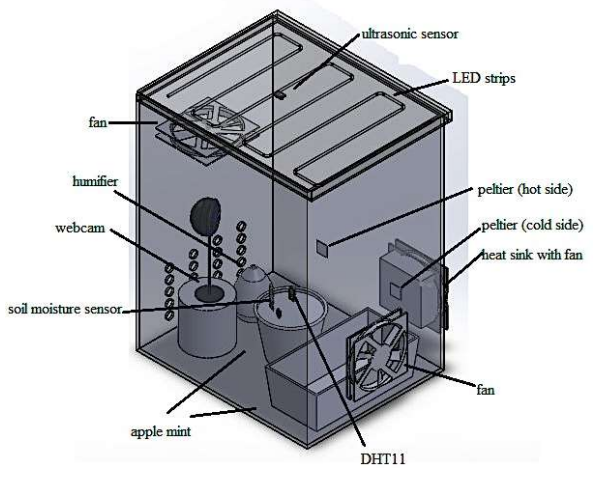

Fig. 3. Implementation of the system.

Figure 3 shows the designed plant growth chamber with the system. The fan on the top is to improve the air ventilation in the chamber where ventilation is an important criteria for plant growth according greenhouse design [9-10]. The surrounding temperature and humidity in this chamber have been detected by DHT11 and stored in MYSQL. The database will then be exported to the excel file and imported to Matlab for graphplot. Figure 4 shows the temperature in the system is within 30 to 35 degree Celsius; meanwhile the relative humidity is maintained at 75 to $90 \%$.

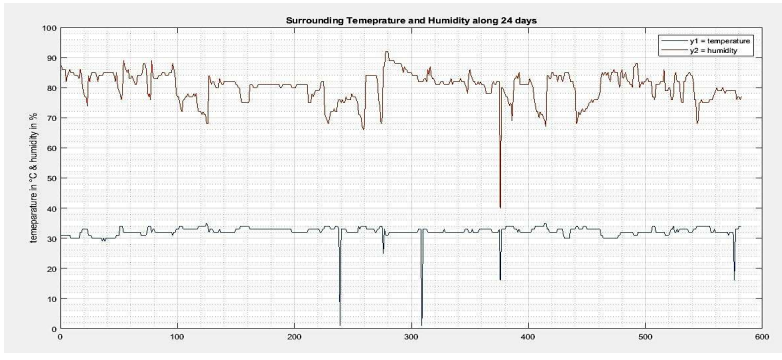

Fig. 4. Temperature and relative humidity in the chamber with the system, top graph is the temperature while bottom graph is the humidity.

Apple mints grown in the system with illumination of artificial light is compared with the one grown outside with illumination of sunlight. After 24 days, they are compared in four aspects, which are the height of the plant, number of leaves, area of the largest leaf of the plant and greenish of the leaves.

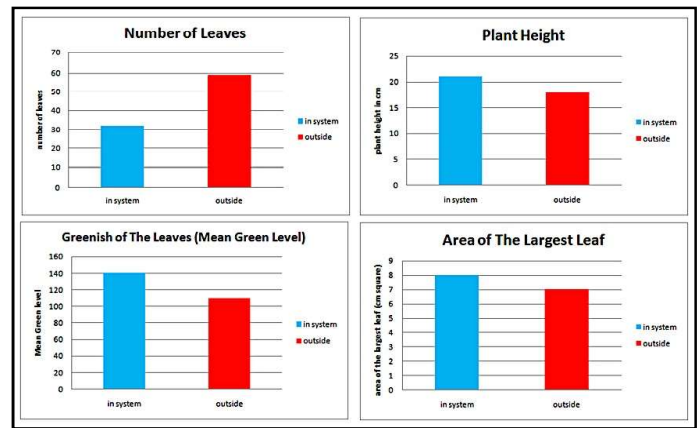

Fig. 5. Comparison of the apple mints in the system and outside the system. 
Figure 5 shows apple mints grown in the chamber with illumination of purple (red+blue)colour, the artificial light is taller, consists of greener leaves and the largest size of leafisfound larger than the apple mints grown under the sunlight. Nevertheless, apple mint grown under the sunlight yield more leaves as compared to the apple mint in the system.

The sensors data such as temperature, humidity and plant height is continuously measured and saved into the database. A webpage created in the local network enables user to view the sensors data. The video of the plant is live streamed in the local network.

\section{Conclusion}

Multicolour LED, the artificial light system has been successfully developed. The Python programmed Raspberry Pi acts as the interface between the sensors, software and others hardware to detect and control the parameters for the better growth of plant. It is proven to be satisfactorily controllable as compared to the intermittent sunlight. Although the system may need further improvement for example consistency of LED intensity, water flow, soil moisture, temperature and humidity, the chosen plant, apple mints are found to grow better in the system. Besides that, far distance real time monitoring on plant is successfully enabled.

\section{References}

1. M.N. Khamis, LED Lighting with Remote Monitoring and Controlling System for Indoor Greenhouse. In: 2017 IEEE Asia Pacific Conference on Postgraduate Research in Microelectronics and Electronics, Malaysia: 81-84. (2017)

2. R.J. Bula, 'Light-Emitting Diodes As A Radiation Source For Plants.' HortScience Journal, 26(2): 203-205. (1991).

3. S.S.S. Yusof, Effect Of Artificial Lighting On Typhonium Flagelliforme For Indoor Vertical Farming. In ProceedingsIEEE International Conference onAutomatic Control and Intelligent Systems (I2CACIS), Malaysia: 7-10. (2017)

4. J. Treder, The Effects Of LEDs On Growth And Morphogenesis Of Vegetable Seedlings Cultivated In Growth Chambers.'In Proceedings2016 IEEE Lighting Conference of the Visegrad Countries (Lumen) at Karpacz, Poland. (2016)

5. E. Danila, Efficient Lighting System For Greenhouses, In Proc. 2016 International Conference and Exposition onElectrical and Power Engineering (EPE), Iasi, Romania: 439-444. (2016)

6. O.E. Hosam, Essential Oils of Mint between Benefits and Hazards, Journal of Essential Oil Bearing Plants. 16(4), 429-438. (2013)

7. H. Wang, Effects Of Light Quality On $\mathrm{CO}_{2}$ Assimilation, Chlorophyll-Fluorescence Quenching Expression Of Calvin Cycle Genes And Carbohydrate Accumulation In Cucumis
Sativus. Journal of Photochemistry and Photobiology B: Biology, 96 (1), 30-37. (2009)

8. Y. Li, Design Of Greenhouse Monitoring And Control System Based On LED Lighting. In 2015 12th China International Forum on Solid State Lighting (SSLCHINA), Shenzhen, China:123-126. (2015)

9. K.S. Kumar, Design And Technology For Greenhouse Cooling In Tropical And Subtropical Regions: A Review.'Energy and Buildings, 41(12), 1269-1275. (2009)

10. S. Kurpaska, Energy Effects During Using the Glass With Different Properties. Journal Technical Sciences / University of Warmia and Mazury in Olsztyn, 17(4), 351-360. (2014) 